\title{
A slowly growing flank mass
}

\author{
Philip W Lam BScPhm MD ${ }^{1}$, David JC Howarth MD FRCPC ${ }^{1,2}$, \\ Anthony Hanbidge MB BCh FRCPC ${ }^{1,2,3}$, Irving E Salit BSC FRCPC $\mathrm{MD}^{1,2,3}$
}

\section{CASE PRESENTATION}

A 62-year-old woman presented to her family physician with a twomonth history of a slowly growing left flank mass. She was previously well except for recurrent urinary tract infections; there was no recent travel or trauma. An ultrasound demonstrated a benign-appearing left renal cyst. Three weeks later, she began to develop flank pain, fevers, chills, night sweats and erythema of the skin over the flank mass. Cultures were not obtained at this time, and she was treated empirically with oral cloxacillin $500 \mathrm{mg}$ four times daily for six weeks with minimal improvement. She developed nausea and vomiting with food consumption, and a $15 \mathrm{~kg}$ weight loss. One month later, the flank mass broke through the skin and began to drain serosanguinous fluid (Figure 1). Review of systems and physical examination were otherwise unremarkable. Initial laboratory investigations demonstrated a normocytic anemia (hemoglobin level $115 \mathrm{~g} / \mathrm{L}$ ), leukocytosis (white blood cell count $15.4 \times 10^{9} / \mathrm{L}$ ), elevated inflammatory markers (C-reactive protein level $171.0 \mathrm{mg} / \mathrm{L}$ and erythrocyte sedimentation rate $85 \mathrm{~mm} / \mathrm{h})$ and a normal creatinine level $(71 \mu \mathrm{mol} / \mathrm{L})$. Computed tomography imaging (Figure 2) revealed a large mass involving the left kidney, encasing other organs on the left side of the abdomen and protruding through the flank. A computed tomography-guided biopsy of the left renal mass was performed.

\section{DIAGNOSIS}

Granulomatous inflammation, von Hansemann cells and MichaelisGuttman bodies (Figure 3) were observed on histopathology; these are characteristic of malacoplakia. Immunohistochemical staining did not support a fungal or malignant etiology. Blood, urine and tissue biopsy cultures grew Escherichia coli. Cultures for mycobacteria and fungi were negative. Her persistent nausea and vomiting was investigated using

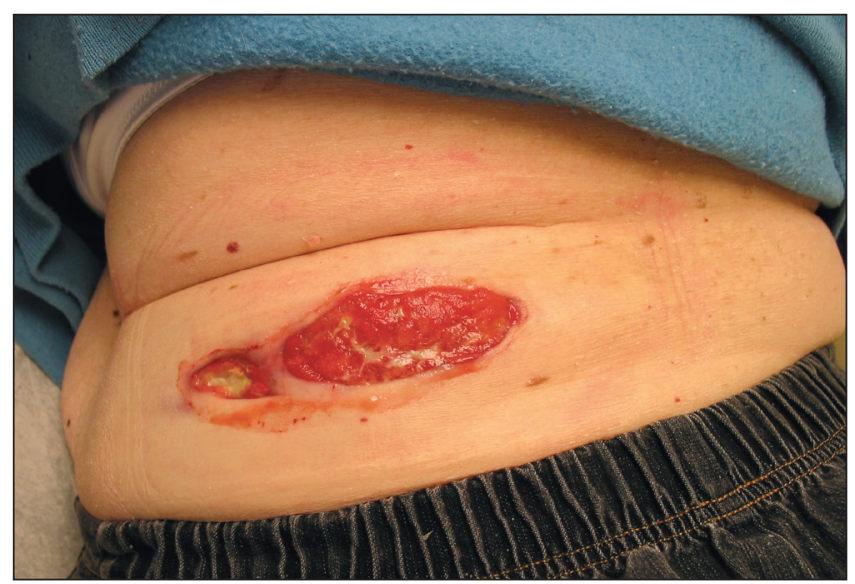

Figure 1) Left-sided flank mass protruding through the skin esophagogastroduodenoscopy, which revealed extension of the renal mass into the fundus of the stomach.

The patient was referred to an infectious diseases specialist and started on oral ciprofloxacin $750 \mathrm{mg}$ every $12 \mathrm{~h}$. Over the next few months, there was a gradual decrease in the size of the mass. The dose of ciprofloxacin was reduced to $750 \mathrm{mg}$ once daily after two months of therapy, and to $500 \mathrm{mg}$ once daily after 10 months of therapy. After a total of 18 months of therapy, the mass had completely regressed, the skin defect had healed and she became asymptomatic with full return of her weight.

\section{DISCUSSION}

Malacoplakia is a rare granulomatous disorder first described by Michaelis and Gutmann in 1902, which classically affects the urinary tract. In one review of 153 cases, urinary tract involvement occurred in $58 \%$ of patients, with the bladder most commonly affected (40\%), followed by the renal parenchyma (16\%) and ureters (11\%) (1). Symptoms of urinary tract malacoplakia include dysuria, frequency and hematuria. With renal involvement, patients can experience fever, flank pain, nausea and a palpable flank mass. Other affected organs can include the gastrointestinal tract, lungs, bone, skin and lymph nodes (2).

Malacoplakia occurs more commonly in females, with a sex predilection of 3:1 (3). Risk factors include immunodeficient states such as diabetes mellitus, immunosuppressive therapy and organ transplantation $(2,3)$. It is strongly associated with E coli urinary tract infection, which is present in $72 \%$ to $87 \%$ of cases $(1,3,4)$.

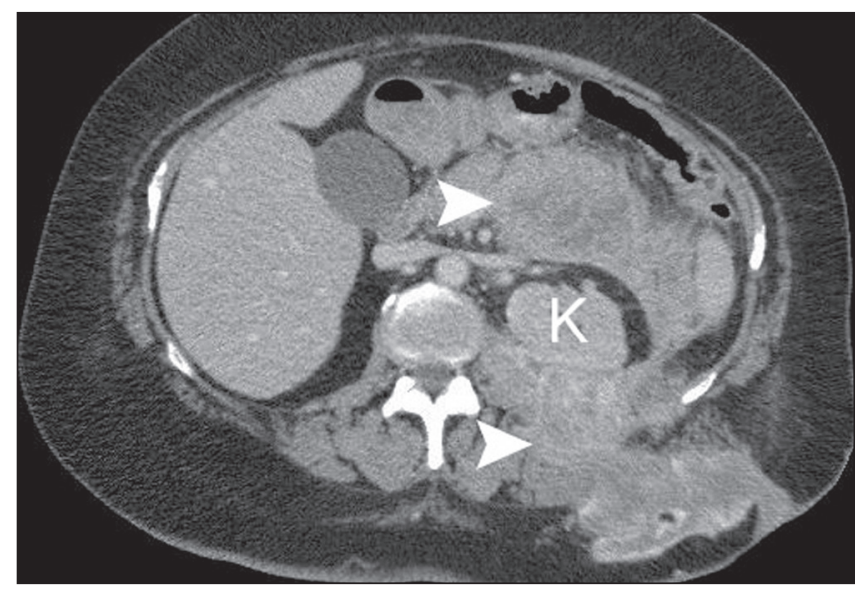

Figure 2) Axial contrast-enhanced computed tomography image through the mid-abdomen showing the left kidney $(K)$ with an infiltrating, heterogeneous inflammatory mass (arrowheads) involving the anterior and posterior pararenal spaces with extension through the posterior abdominal wall and skin

\footnotetext{
${ }^{1}$ University of Toronto; ${ }^{2}$ Mount Sinai Hospital; ${ }^{3}$ University Health Network, Toronto, Ontario

Correspondence: Dr Irving E Salit, Toronto General Hospital, 200 Elizabeth Street, 13 Eaton North - Room 215, Toronto, Ontario M5G 2C4.

Telephone 416-340-3697, fax 416-340-3357, e-mail irving.salit@uhn.ca
} 


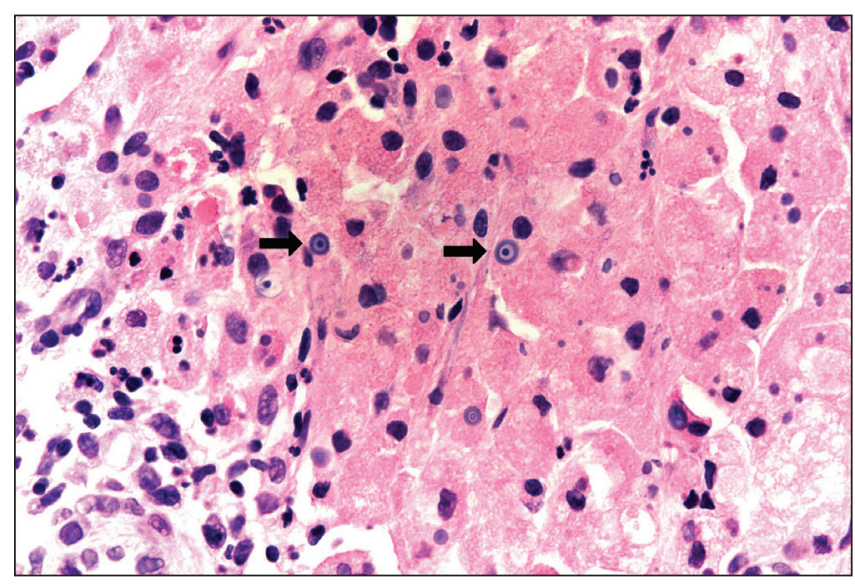

Figure 3) Renal mass biopsy section (hematoxylin and eosin stain, original magnification $\times 400$ ) demonstrating collections of large histiocytes with granular eosinophilic (pink) cytoplasm (von Hansemann cells) and Michaelis-Gutmann bodies (arrows)

Malacoplakia is believed to occur because of impaired phagocytosis by macrophages and defective intracellular bacterial killing. This abnormality has been linked to low intracellular levels of cyclic GMP, resulting in decreased microtubule formation, beta-glucuronidase release and phagolysosome digestion (5). In one report, E coli grown from bladder malacoplakia tissue was identical to E coli grown from the patient's urine culture six months previously (6). This suggests that the persistence of urinary tract pathogens due to impaired phagocytic activity may contribute to the development of malacoplakia (6).

The radiographic appearance of renal malacoplakia includes nephromegaly with unifocal or multifocal solid masses, and can appear similar to xanthogranulotamous pyelonephritis or renal cell carcinoma (2); biopsy of the affected tissue is essential for confirming the diagnosis. Histological examination of malacoplakia-affected tissue demonstrates granulomatous infiltration with large eosinophilic macrophages (von Hansemann cells) (6). Bacteria ingested by the macrophages are not completely destroyed and persist within phagolysosomes. These structures become mineralized, giving the characteristic intracellular calcified 'bulls-eye' lamellar inclusions (termed Michaelis-Gutmann bodies) which stain strongly with periodic acid-Schiff (6). Michaelis-Gutmann bodies are pathognomonic for malacoplakia.

Treatment is focused on improving phagocytosis and intracellular killing of bacteria. Ciprofloxacin and trimethoprim/sulfamethoxazole (TMP/SMX) are most commonly used due to their bactericidal activity against coliforms, and ability to penetrate leukocytes and phagolysosomes (2). In one review of treatment in 140 cases of malacoplakia, patients receiving quinolone therapy alone $(n=10)$ demonstrated a $90 \%$ cure rate, compared with $21 \%$ in those receiving nonquinolone therapy alone $(n=19)(7)$; all patients who achieved cure using nonquinolone therapy had received TMP/SMX (7). Current resistance rates suggest that TMP/SMX is inferior and should only be used when in vitro activity has been demonstrated. There are currently no guidelines for the treatment of malacoplakia; thus, the therapy and duration is based on susceptibilities, individual clinical response and the known effectiveness of ciprofloxacin.
Adjunctive therapy with bethanechol (an anticholinergic agent) and ascorbic acid are believed to enhance leukocyte intracellular killing activity by increasing intracellular cyclic GMP (6), but their efficacy has not been rigorously tested (7). For immunocompromised individuals, such as renal transplant patients, discontinuation or dose reduction of immunosuppressive agents may result in resolution of the malacoplakia (7).

Surgical intervention to treat malacoplakia has ranged from endoscopic resection of bladder lesions to complete nephrectomy (2). The need for surgical intervention is dependent on the clinical status, location and extent of disease, presence of urinary obstruction and failure of medical therapy.

Bilateral renal malacoplakia has been associated with increased morbidity and mortality: in one review of cases from 1981 to 1993, the mortality rate was $80 \%$ in those treated with non-leukocyte-penetrating antibiotics or with surgery (2). In those treated with optimal antibiotics (ciprofloxacin or TMP/SMX), the mortality rate from bilateral renal malacoplakia was $22 \%$ (2). A review of 25 renal malacoplakia cases reported after 1990 demonstrated a survival rate of 92\%, with $70 \%$ receiving medical treatment only (4). Even with effective antibiotic therapy, patients with significant parenchymal destruction may develop renal insufficiency and require dialysis. Thus, prompt diagnosis of renal malacoplakia and institution of proper antimicrobial therapy is essential in minimizing long-term sequelae.

\section{SUMMARY}

We presented a case of renal malacoplakia in a woman with a history of recurrent urinary tract infections. The extent of contiguous spread was remarkable, with involvement of the surrounding organs and skin. The clinical and radiological presentation of malacoplakia is nonspecific and ultimately requires a biopsy for definitive diagnosis. Our patient was cured after 18 months of oral fluoroquinolone therapy, supporting the documented efficacy of leukocyte-penetrating antibiotics as the mainstay of therapy.

\section{REFERENCES}

1. Stanton MJ, Maxted W. Malacoplakia: A study of the literature and current concepts of pathogenesis, diagnosis and treatment. J Urol 1981;125:139-46.

2. Mitchell MA, Markovitz DM, Killen PD, Braun DK. Bilateral renal parenchymal malacoplakia presenting as fever of unknown origin: Case report and review. Clin Infect Dis 1994;18:704-18.

3. Dobyan DC, Truong LD, Eknoyan G. Renal malacoplakia reappraised. Am J Kidney Dis 1993;22:243-52.

4. Tam VK, Kung WH, Li R, Chan KW. Renal parenchymal malacoplakia: A rare cause of ARF with a review of recent literature. Am J Kidney Dis 2003;41:E13-7.

5. Abdou NI, NaPombejara C, Sagawa A, et al. Malacoplakia: Evidence for monocyte lysosomal abnormality correctable by cholinergic agonist in vitro and in vivo. N Engl J Med 1977;297:1413-9.

6. Lewin KJ, Fair WR, Steigbigel RT, Winberg CD, Droller MJ. Clinical and laboratory studies into the pathogenesis of malacoplakia. J Clin Pathol 1976;29:354-63.

7. van der Voort HJ, ten Velden JA, Wassenaar RP, Silberbusch J. Malacoplakia. Two case reports and a comparison of treatment modalities based on a literature review. Arch Intern Med 1996;156:577-83. 


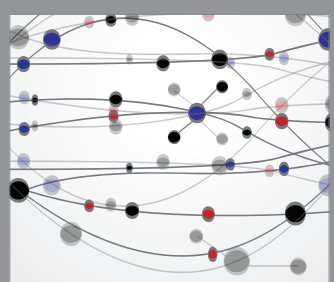

The Scientific World Journal
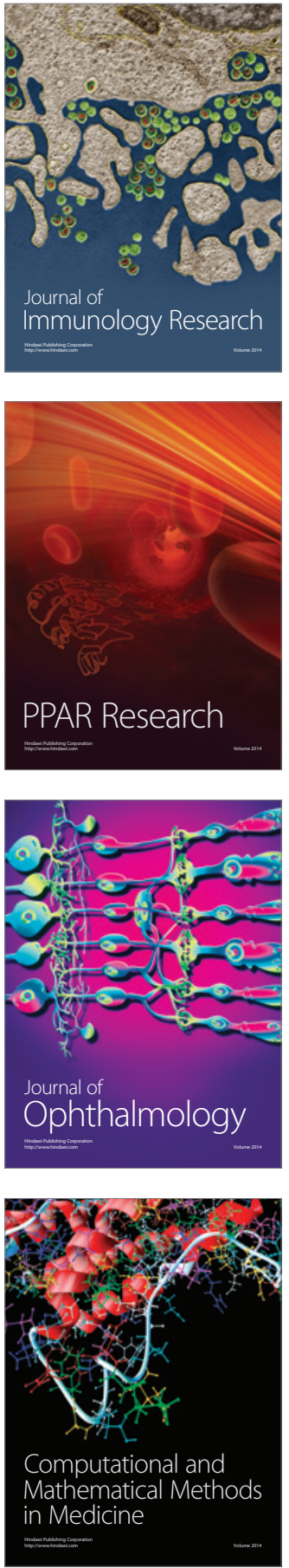

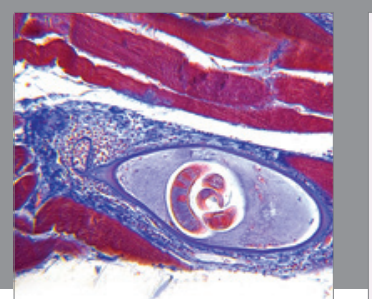

Gastroenterology Research and Practice

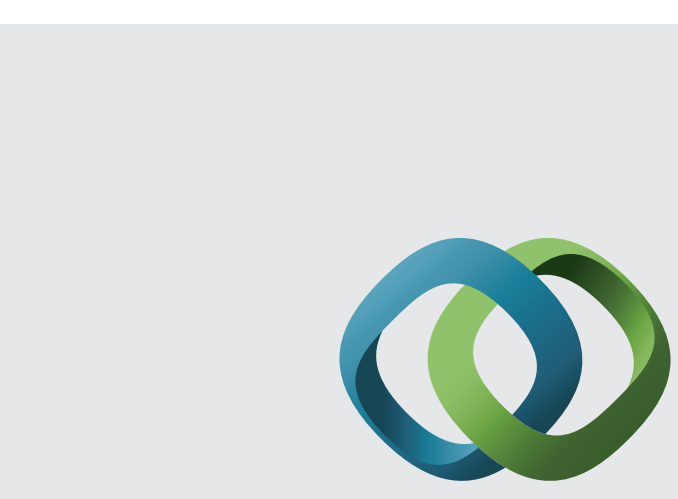

\section{Hindawi}

Submit your manuscripts at

http://www.hindawi.com
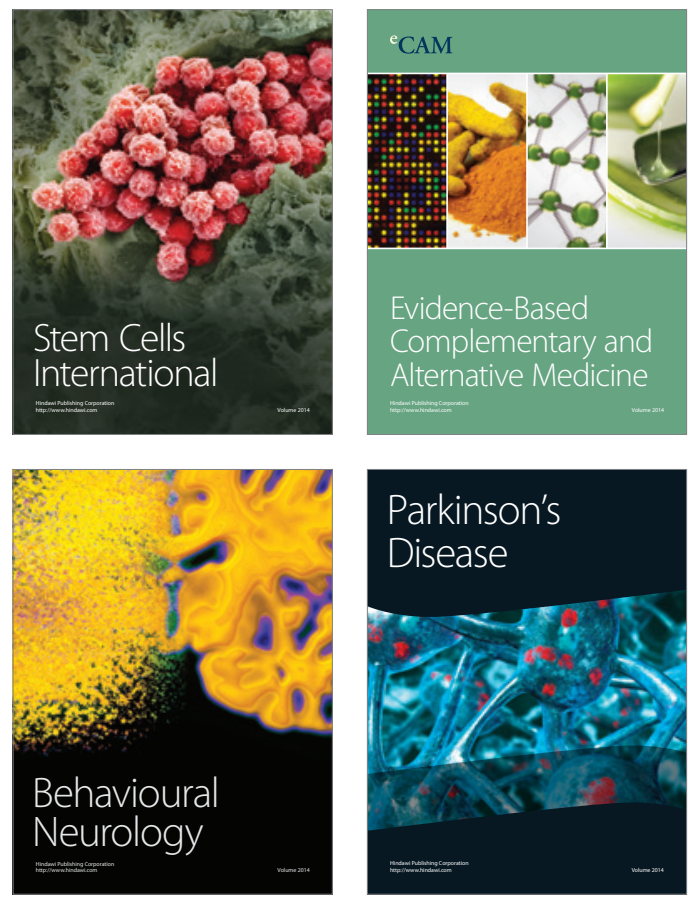
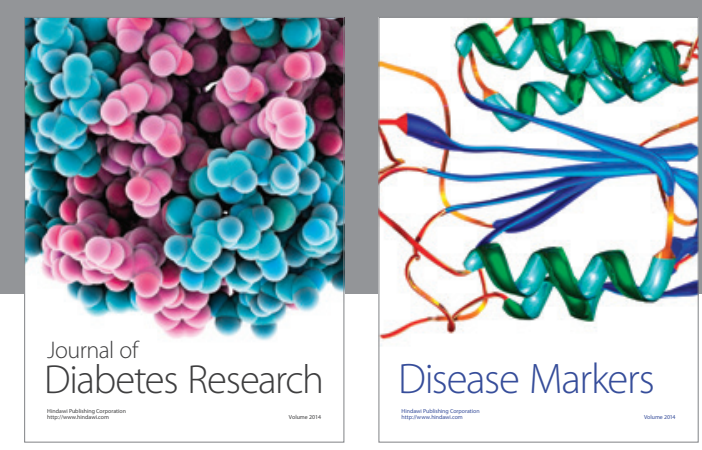

Disease Markers
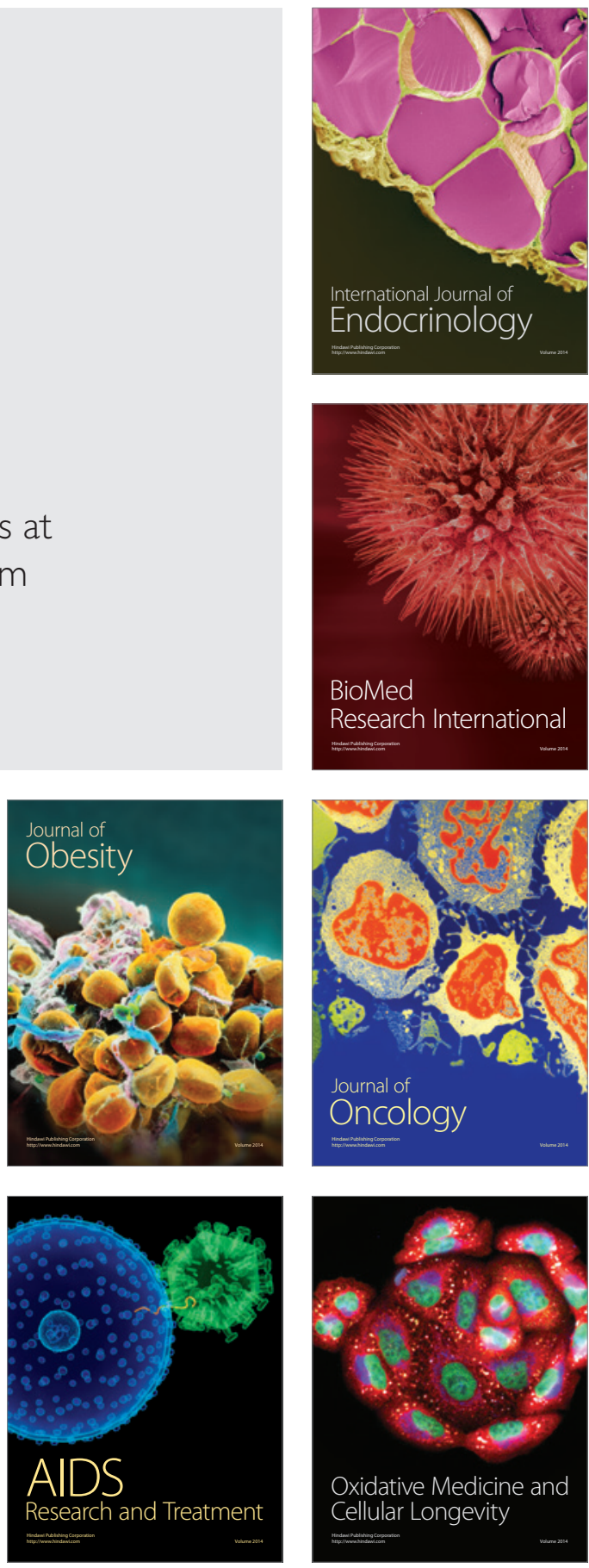